\title{
Zebrafish phenotypic screen identifies novel Notch antagonists
}

\begin{abstract}
Zebrafish represents a powerful in vivo model for phenotype-based drug discovery to identify clinically relevant small molecules. By utilizing this model, we evaluated natural product derived compounds that could potentially modulate Notch signaling that is important in both zebrafish embryogenesis and pathogenic in human cancers. A total of 234 compounds were screened using zebrafish embryos and 3 were identified to be conferring phenotypic alterations similar to embryos treated with known Notch inhibitors. Subsequent secondary screens using HEK293T cells overexpressing truncated Notch1 (HEK293T $\Delta$ E) identified 2 compounds, EDD3 and 3H4MB, to be potential Notch antagonists. Both compounds reduced protein expression of NOTCH1, Notch intracellular domain (NICD) and hairy and enhancer of split-1 (HES1) in HEK293T $\Delta \mathrm{E}$ and downregulated Notch target genes. Importantly, EDD3 treatment of human oral cancer cell lines demonstrated reduction of Notch target proteins and genes. EDD3 also inhibited proliferation and induced G0/G1 cell cycle arrest of ORL-150 cells through inducing p27KIP1. Our data demonstrates the utility of the zebrafish phenotypic screen and identifying EDD3 as a promising Notch antagonist for further development as a novel therapeutic agent.
\end{abstract}

Keyword: Notch signalling; Zebrafish; Phenotypic assay; Natural products; Human oral cancer 